\title{
A Beamforming Scheme with Steering and Nulling Capabilities for Direct Radiating Array Based Satellite Antenna
}

\author{
Xiao GENG \\ Institute of Advanced Communications \\ Peking University \\ Beijing, China \\ e-mail: gengxiao@pku.edu.cn \\ Yilei SUN \\ School of Franchise Management \\ Beijing Normal University (Zhuhai Campus) \\ Beijing, China
}

\author{
Yong LUO \\ Institute of Advanced Communications \\ Peking University \\ Beijing, China \\ Yuxin CHENG \\ Institute of Advanced Communications \\ Peking University \\ Beijing, China
}

\author{
Jianjun WU \\ Institute of Advanced Communications \\ Peking University \\ Beijing, China \\ e-mail: just@pku.edu.cn
}

\begin{abstract}
In the current satellite communication systems, the multi-beam satellite system has been widely used. This paper analyses the typical fixed multi-beam system and designs a simulation program called DRA for beam forming. The beam can have any directivity, and can eliminate interference with the null steering in a specific direction of the beam coverage, enabling users to communication with a beam in the satellite coverage.
\end{abstract}

Keywords-satellite communication; multi-beam forming algorithm; null steering algorithm; satellite communication; multi-beam forming algorithm; null steering algorithm

\section{INTRODUCTION}

Compared with other satellite communication systems, the LEO satellite communication system has its own characteristics: low latency, low path loss, high user experience, and it can be used to communicate with a small terminal, without the high sensitivity of high orbit satellite handsets.

In the present low earth orbiting (LEO) satellite communication system, multi-beam satellite system has been widely used. Now in the most of the multi beam satellite communication systems, the fixed multi beams are used to communicate with the users. When the satellite is moving, the wave beam needs to be switched frequently[1]. So the users possess too many resources. And the nulls in the coverage area need to be formed to suppress interferences[2]. The nulling methods are generally based on controlling the amplitude only [3], or the phase-only [4]. The major points of the paper research are: Any beam of direction can be synthesized, based on feed horn source and triangular grid direct radiating array (DRA) in the low earth orbiting satellite communication system, and when covered by the satellites, users maintain to communication with a beam; When there is a strong interference in the service area, the beamforming scheme is proposed with null steering to inhibit the interferences.

The paper is organized as follows. The horn feed radiation field is shown in Section 2 and A DRA beam-forming scheme is shown in Section 3. Numerical analysis and discussion for results are stated in Section 4.The conclusion is developed in Section 5.

\section{GENERAL STRUCTURES}

The conical horn has a very complex radiation field. To simplify the problem, when the conical horn radiation field is calculated, there is limit to the conical horn[5].

1) The main mode in the conical horn field is. The amplitude distribution of the surface field is equivalent to the circular waveguide surface.

2) Do not consider the reflected wave at the mouth of the conical horn field.

3) Ignore the current and higher modes which outflow from the horn mouth.

According to the limit, introduced by the Maxwell equation, the function relation of the far field distribution of the conical horn is given by:

$$
E_{p}=-\frac{1}{\varepsilon_{0}} \nabla \times F-\frac{j}{\omega \mu_{0} \varepsilon_{0}} \nabla \times \nabla \times A
$$


The formula for the far field radiation of the conical horn is [5]:

$\left(E_{p}\right)_{\theta}=\frac{-j k}{4 \pi} \frac{e^{-j k R}}{R}(1+\cos \theta)\left(N_{x} \cos \varphi+N_{y} \sin \varphi\right)=D_{11} \frac{e^{-j k R}}{R}\left(\frac{1+\cos \theta}{2}\right)\left(I_{2}-I_{1}\right) \sin \varphi$

$\left(E_{p}\right)_{\varphi}=\frac{-j k}{4 \pi} \frac{e^{-j k R}}{R}(1+\cos \theta)\left(N_{x} \sin \varphi-N_{y} \cos \varphi\right)=D_{11} \frac{e^{-j k R}}{R}\left(\frac{1+\cos \theta}{2}\right)\left(I_{2}+I_{1}\right) \cos \varphi$

So the far field formula for radiation intensity is:

$$
E_{p}=F_{e}(\theta, \varphi)=\sqrt{\left(E_{p}\right)_{\theta}^{2}+\left(E_{p}\right)_{\varphi}^{2}}
$$

Considering the relationship of low orbit antenna array and frequency, the horn parameters are used as shown below:

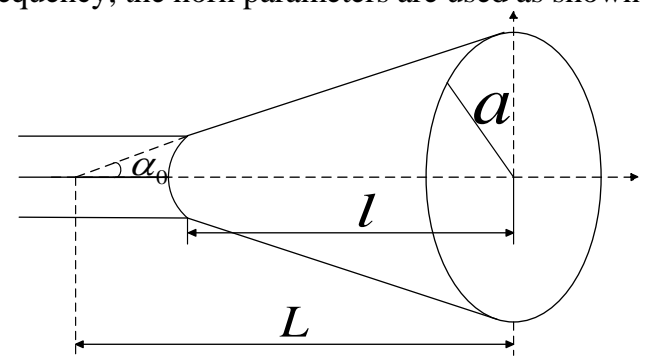

Figure 1. The horn feed source

TABle I. The Parameters Table of Horn Antenna FeEd Source

\begin{tabular}{|c|c|c|}
\hline & Description & Value \\
\hline$a(\mathrm{~m})$ & Bell mouth radius & 0.0675 \\
\hline$L(\mathrm{~m})$ & The total length of horn waveguide & 0.202 \\
\hline$l(\mathrm{~m})$ & Physical length & 0.158 \\
\hline$\alpha_{0}\left({ }^{\circ}\right)$ & Bell mouth opening Angle & 19.5 \\
\hline
\end{tabular}

Adjusting the feed distance in uniform planar array, we can get the planar array as shown in Fig. 2. It's supposed that there is a triangular grid array which is composed by $\mathrm{N}$ isotropic units whose distances are $a$ to the center. Generally, the array is located in the xoy plane. The radiation formula in the space of the array is [6]:

$F(\theta, \varphi)=\mathrm{F}_{e}(\theta, \varphi) \mathrm{S}_{3}(\theta, \varphi)=\sqrt{\left(E_{p}\right)_{\theta}^{2}+\left(E_{p}\right)_{\varphi}^{2}} \sum_{n=1}^{N} \dot{I}_{n} \exp \left[j k a_{n} \sin \theta \cos \left(\varphi-\beta_{n}\right)\right]$

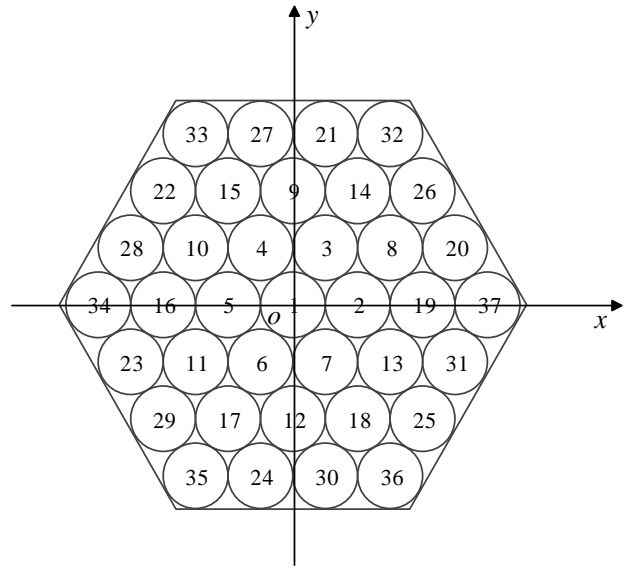

Figure 2. The distribution diagram of the horn feed sources

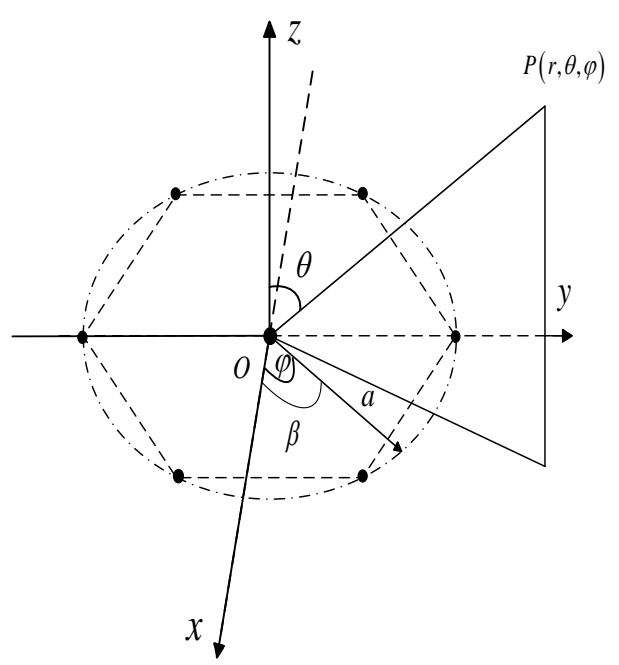

Figure 3. The triangular lattice array figure

As Fig. 3 shown, the horn radius is $a$, and each feed radius is given by:

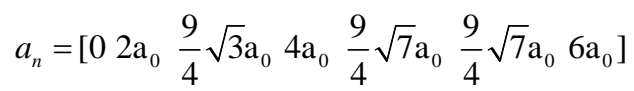

According to the initial phase $\beta_{0}=[0, \mathrm{pi} / 3,2 \mathrm{pi} / 3, \mathrm{pi}, 4 \mathrm{pi} / 3,5 \mathrm{pi} / 3], a_{0}=a^{*}$ ones $(1,6)$, the phase formulation with feed source is calculated as:

$\beta_{n}=\left[0, \beta_{0}, \beta_{0}+\mathrm{pi} / 6, \beta_{0}, \beta_{0}+\mathrm{pi} / 6-\operatorname{actan}(1 / 3 \sqrt{3}), \beta_{0}+\mathrm{pi} / 6+\operatorname{actan}(1 / 3 \sqrt{3}), \beta_{0}\right]$

Given $F\left(\theta_{3 d B}, 0\right)=0.707 F(\theta, 0)$, the $3 \mathrm{~dB}$ beam angle for 37 feed source in the longitudinal direction is 1.02 degrees. 


\section{DRA BEAMFORMING SCHEME}

\section{A. Analysis of Arbitrary Beam Directivity}

As shown in Fig. 3, the triangular lattice planar array is weighted according to the target. In $\dot{I}_{n}=I_{n} e^{j \alpha_{n}}, I_{n}$ is the incentive current amplitude of unit n, and $\alpha_{n}$ is the incentive phase that the center of the array is the reference point. With total effect that feed sources contribute to the radiant far field, the function of radiant far field is:

$$
F(\theta, \varphi)=\sqrt{\left(E_{p}\right)_{\theta}^{2}+\left(E_{p}\right)_{\varphi}^{2}} \sum_{n=1}^{N} I_{n} \exp \left[j k a_{n} \sin \theta \cos \left(\varphi-\beta_{n}\right)+j \alpha_{n}\right]
$$

$$
\alpha_{n}=-k a_{n} \sin \theta_{i} \cos \left(\varphi_{i}-\beta_{n}\right)
$$

$\theta_{i}, \varphi_{i}$ are the constants that can get the space radiation of the target. It's assumed that the radiation pattern is $\mathrm{S}_{0}\left(\theta_{0}, \varphi_{0}\right)$, and $\theta_{0}, \varphi_{0}$ are the angles of the direction. The radiation pattern which is weighted is reaching the target pattern.

To simplify the calculation, the radiation pattern is the same as the ideal pattern. It means, $\min \left|\mathrm{S}(\theta, \varphi)-\mathrm{S}_{0}\left(\theta_{0}, \varphi_{0}\right)\right|$ is equal to zero. So the incentive phase is:

$$
\alpha_{n}=-k a_{n} \sin \theta_{0} \cos \left(\varphi_{0}-\beta_{n}\right)
$$

The formula of the radiation is:

$F(\theta, \varphi)=\sqrt{\left(E_{p}\right)_{\theta}^{2}+\left(E_{p}\right)_{\varphi}^{2}} \sum_{n=1}^{N} I_{n} \exp \left[j k a_{n} \bullet \sin \theta \cos \left(\varphi-\beta_{n}\right)-j k a_{n} \sin \theta_{0} \cos \left(\varphi_{0}-\beta_{n}\right)\right.$

And the weighting factor is:

$$
w_{n}=I_{n} \exp \left(-j k a_{n} \sin \theta_{0} \cos \left(\varphi_{0}-\beta_{n}\right)\right)
$$

\section{B. The Algorithms for Shape Preserving and Nulling Adjustment of Beam}

There is an important question in the multi-beam synthesis that the null steering is formed in the position of interfering, and the communication is normal in the areas of the other direction. The algorithm based on the thought is the null steering with conformal condition. So the equation based on the algorithm is as follow:

$$
\left\{\begin{array}{c}
\min (\Delta \varepsilon)=\min \left\{\left|w-w_{q}\right|\right\} \\
\min \left(w^{H} C\right)
\end{array}\right.
$$

$w_{q}$ is the beamforming weight vector when there is not interfering. And $w$ is the weight vector of null steering. $C$ is the normalized unit direction matrix in the interfering direction. The zero weight vector can be got based on the conformal algorithm, with the Lagrange multiplier method.

$$
w_{\text {null }}=\left(I-C\left(C^{H} C\right)^{-1} C^{H}\right) w_{q}
$$

In the process of satellite mobile communication, outside the target radiation direction, for some sensitive areas we don't want them to get the energy signal or to be disturbed. Therefore, the multi-beam synthesis algorithm is used. The main lobe of the array is close to the target along with the null steering pointing to the interference direction. According to the requirements it can be transformed into the following optimization problem:

$$
\left\{\begin{array}{rr}
\min \left\|w-w_{q}\right\|^{2} \\
\text { s.t } & w^{H} C=f
\end{array}\right.
$$

The optimal power of the Lagrange multiplier is:

$w_{\text {opt }}=w_{q}-C * \operatorname{inv}\left(C^{H} * C\right) * C^{H} * w_{q}-C * i n v\left(C^{H} * C\right) * f$

The longitudinal dimension of $C$ is the direction vector of the interference direction. $f$ is the constraint value vector. Mainly to the inhibition of null steering direction, so $f$ is the null direction.

How to ensure the radiation direction of the target is important under the impact of the interference. In this section conformal algorithms are applied, and the constraints are increased, to ensure zero in the direction of the interference.

The constraints are formed between the new weight vector and the static weight vector.

$$
\min \left\|w-w_{q}\right\|^{2}
$$

The radiation direction is towards the target direction, and at the same time there are constraints:

$$
w^{H} C=f
$$

The increment caused by a new weight vector is zero in the direction of the interference. It means that the 
shape-preserving of the target beams is completed, and the zeroing in the direction of the inference is also realized. What's more? It can be seen that scanning beams of any direction can be implemented, with the adjustment of the static weight vectors at the same time. The zero adjustment from one to many directions can be achieved with the adjustment of the interfering matric $\mathrm{C}$.

\section{DRA BEAMFORMING SCHEME}

When $\theta_{0}, \varphi_{0}$ point to $\left(2^{\circ}, 160^{\circ}\right)$ and $\left(8^{\circ}, 80^{\circ}\right)$, the radiation distribution in the range of the $3 \mathrm{~dB}$ beam coverage within 10 degrees in the service area is shown in the following figures.

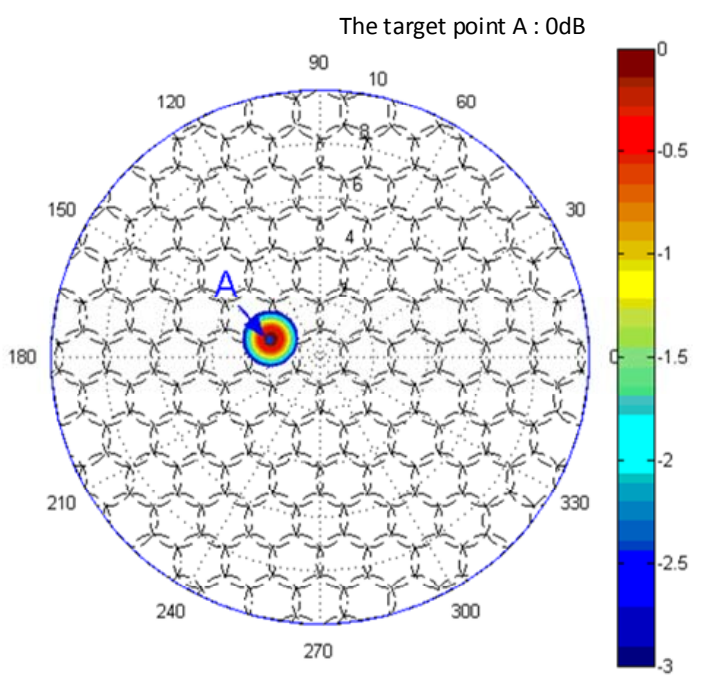

(a) The angle is $\left(2^{\circ}, 160^{\circ}\right)$

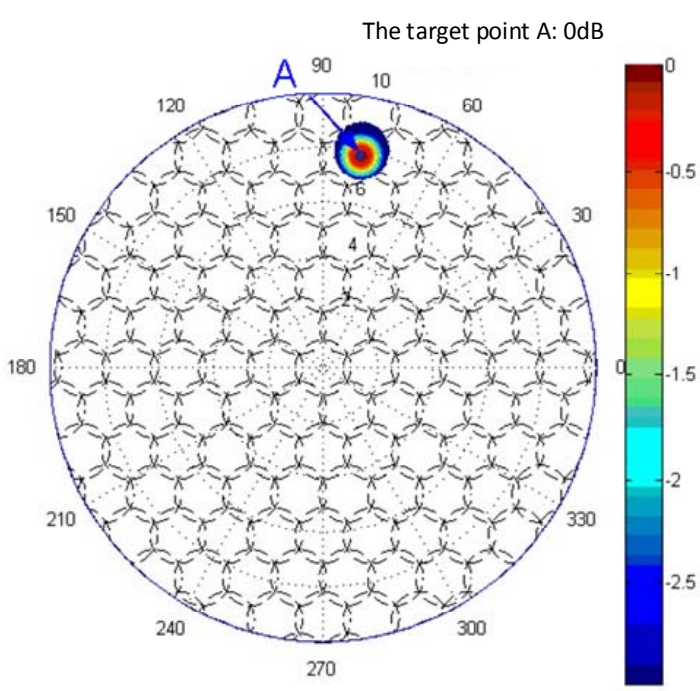

(b) The angle is $\left(8^{\circ}, 80^{\circ}\right)$

Figure 4. The $3 \mathrm{~dB}$ beam distribution of 37 feed sources
When the target radiation angles are $\theta_{0}, \varphi_{0}$.Combined with classical beamforming algorithms, change the weighting factors $\theta_{i}, \varphi_{i}$ to the direction.

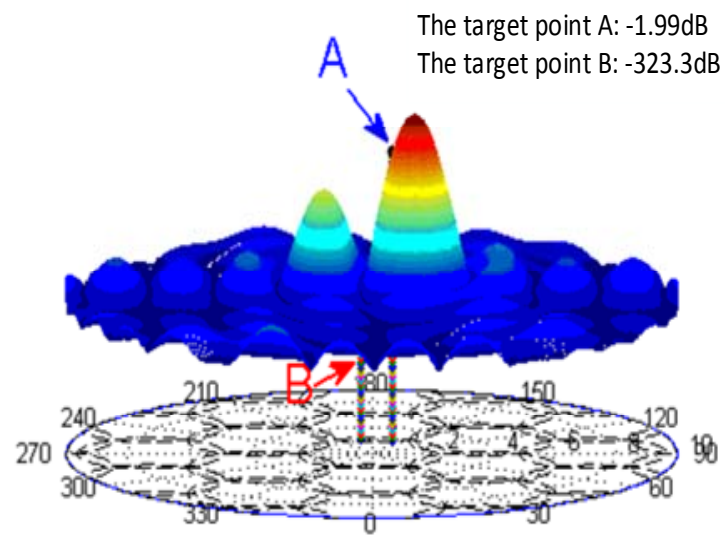

Figure 5. The space diagram of null steering of 37 feed sources

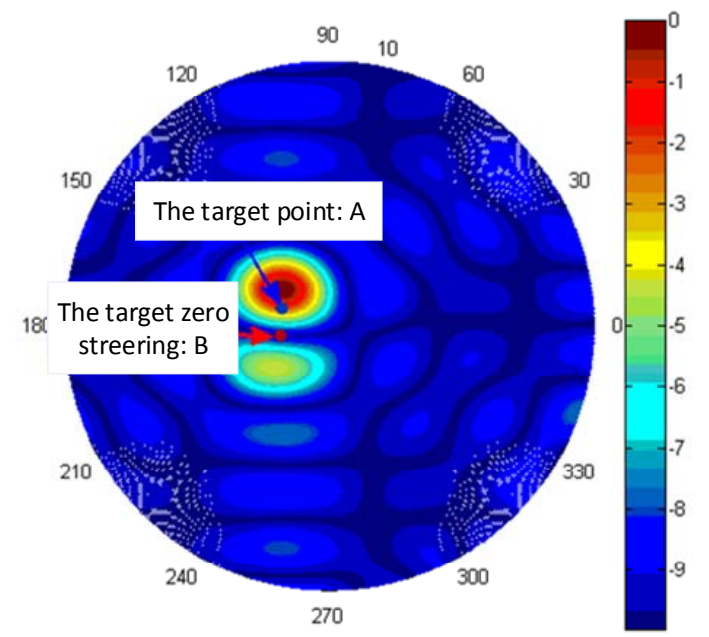

Figure 6. The planform diagram of nulldistribution steering distribution of 37 feed sources

Shown by Fig. 5 and Fig. 6, it appears to interfere in the direction of the beam coverage. The algorithms of adjusting zero and shape preserving are used to suppress the interference. The depth of nulling is $323.3 \mathrm{~dB}$, achieving the inhibition effect. At the same time, the radiation intensity of the target is maintained at $-1.99 \mathrm{~dB}$, which meets the requirements of the radiation.

\section{SUMMARY}

According to the feed horn and triangular grid direct radiating array, a scheme to form the arbitrary directional beam in the covering range is proposed. Users in the coverage of the satellite, can keep to communication with a beam. This kind of communication mode can reduce the signal resources and maintain the stability of the service quality. And when there is a strong interference in the service area, the algorithm 
of null steering is used to inhibit the interference. Numerical calculation and analysis are taken to demonstrate the scheme.

\section{ACKNOWLEDGEMENTS}

This work is supported by the National Natural Science Foundation of China (No.61371073) . Corresponding author: Jianjun Wu; Phone: +86-10-62752848; Email: just@pku.edu.cn.

\section{REFERENCES}

[1] Crowe, K.E. A model to describe the distribution of transmission path elevation angles to the Iridium and Globalstar satellite systems. Communications Letters, IEEE Vol. 3, Issue 8
[2] Steyskal, H., R. A. Shore, and R. L. Haupt, "Methods for null control and their effects on the radiation pattern," IEEE Trans. Antennas Propagat., Vol. 34, 404-409, 1986.

[3] Babayigit, B., A. Akdagli, and K. Guney, "A clonal selection algorithm for null synthesizing of linear antenna arrays by amplitude control," Journal of electromagnetic Waves and Applications, Vol. 20, 1007-1020, 2006.

[4] Haupt, R. L., "Phase-only adaptive nulling with a genetic algorithm," IEEE Trans. Antennas Propagat., Vol. 45, 1009-1015, 1997.

[5] Narasimhan, B.V., Models in a conical horn: new approach. Proc. IEE. Vol. 118, NO. 2.

[6] Xue Zhenghi, Li Weiming, Ren Wu., Antenna array analysis and synthesis. Beihang University Press. 2011. 